\title{
Requirements for a Control Instrument of Intraorganizational Online Collaboration - A Maturity Model Analysis
}

\author{
Samuel Reeb \\ TU Dresden \\ $\underline{\text { samuel.reeb@ }}$ \\ $\underline{\text { tu-dresden.de }}$
}

\author{
Franziska Dilefeld \\ TU Dresden \\ franziska.dilefeld@ \\ $\underline{\text { mailbox.tu-dresden.de }}$
}

\author{
Philip Schebesta \\ TU Dresden \\ philip.schebesta@ \\ mailbox.tu-dresden.de
}

\author{
Johanna Szabó \\ TU Dresden \\ johanna.szabo@ \\ mailbox.tu-dresden.de
}

\begin{abstract}
Intraorganizational online collaboration (IOC) can be designed in various ways but there is still a backlog in how to control IOC and deriving corresponding actions. This paper aims to find and analyze approaches for an evaluation model of intraorganizational online collaboration. By using interview data, of in-depth interview with field experts the importance of an organizational control instrument for IOC is elaborated and a requirement catalog for such instruments is deduced. This catalog is applied in an initial analysis of maturity models (MM) as one identified approach of a control instrument for IOC. The findings show that the analyzed MMs fulfil the catalog of requirements in different degrees and that suitable approaches exist. However, all MMs do have disadvantages and further developments of the models are required.
\end{abstract}

\section{Introduction}

Globalization and the accompanying digital transformation are two of the main drivers and simultaneously challenges of the modern world, which are changing societies' general conditions [41]. Geographical distances are becoming less important due to new technologies. This opens new possibilities for distributed organizations and teams as well as people working remotely. Consequently, there is a vast scope of research on approaches, methods, and actions to design intraorganizational online collaborative work available $[8,21]$. Organizational control instruments to asses and manage intraorganizational online collaboration (IOC) are only of minor interest in current research $[10,15]$.

However, to design and maintain IOC effectively and efficiently, an organizational control instrument is indispensable [18]. Control instruments provide a more objective insight into the organization's situation and help to identify problem areas. Nonetheless, a study from 2016 shows that only $13 \%$ of the surveyed organizations use control instruments for IOC at an organizational level [4]. Depending on the control instrument's domain, there are different requirements.

This research explores control instruments for IOC with a focus on the organizational level of business organizations, their relevance and practical usability. Therefore, it examines what requirements exist for an IOC control instrument from a theoretical and practical perspective for business organizations. We consider Maturity Models (MM) to be a suitable control instrument for IOC, as they meet the process character of IOC [3, 35]. Furthermore, the qualitative characteristics of IOC are adequately addressed by MMs, reducing biases caused by explicit numerical values $[25,30]$. Therefore, as an initial approach, existing MMs for collaborative work are analyzed regarding the practical requirements.

The research design follows Peffers [32] and intends to develop and apply a design artifact of a requirement catalog for an organizational control instrument for IOC. First the theoretical and practical relevance of this endeavor are elaborated and the artifact's objectives are defined [32]. The artifact aims to provide a set of requirements to evaluate an organizational control instrument, regarding its goodness of fit to design and maintain IOC. Therefore, this paper illustrates the relevance of an organizational control instrument for IOC, identifies practical requirements for such an instrument and demonstrates their applicability, by applying it to the MM context. To this end the following two research questions will be addressed:

- What are the practical requirements for an organizational control instrument for IOC?

- To what extent do MMs for collaboration meet the practical requirements?

To define the problem situation from the rigorous side, section two presents the background and related organizational control instruments. Thereby, MMs are identified as a suitable approach for a control instrument for IOC from the theoretical perspective. To confirm the practical relevance of an organizational control 
instrument for IOC and to identify its requirements, semi-structured in-depth interviews were conducted. To demonstrate the applicability of the requirement catalog, it was applied to MMs for collaboration. In section four the results are presented, with the results of the application of the requirement catalog discussed in section five. Concluding implications for further research are given.

\section{Conceptual Background}

An initial search regarding online collaboration showed that a vast variety of terms exists in the literature referring to the same concept of working together. Exemplary terms are virtual collaboration, ecollaboration, smart collaboration and web 2.0 collaboration. Collaboration describes a process in that at least two individuals work together through communication and interaction to achieve a common understanding of a product, process or event [35]. To distinguish this from the concept of coordination it is useful to have a look at the component of synchronicity. The procedure of coordination consists of dividing, assigning and independent processing of tasks and combining these into a joint final product [17]. Whereas collaboration is an interactive process, where individuals synchronously coordinate and accomplish work together $[2,17]$.

This paper focuses on collaboration in an intraorganizational as well as an online context. The virtual space allows people to work together over large distances. Although the collaboration happens in a virtual setting, the processes and their output are nevertheless real [45]. Furthermore, collaboration can exist on different levels, e.g. between teams or organizations. This paper deals exclusively with online collaboration within business organizations. Therefore, IOC describes an interactive process of synchronously coordinated work in a virtual environment towards common goals within an organization.

Distributed organizations and teams are not just working in different locations but must collaborate through geographical distance. They differ from colocated ones in more than just their geographical allocation, but also in aspects as trust building [26], leadership [39] and the development of a shared culture [11]. Regardless of team or organization type, effective and efficient forms of communication are of critical relevance for good collaboration [15]. Thus, it must become an actively managed part of the organization.

Prior scientific research has highlighted the importance of organizational control as a crucial requirement of effective and efficient management of the organization [10]. Organizational control describes mechanisms used by the organization and/or managers to guide organizational units and/or employees regarding an effective and efficient resource allocation to achieve business objectives [27, 40]. Therefore, organizational control instruments indicate improvement potentials and trigger actions to achieve the defined goals [20].

An organizational control system encompasses a configuration of organizational control instruments [37] with three main functions:

- the control mechanisms are linked to business objectives (CR1)

- the control mechanisms give the organization and/or managers decision support (CR2)

- the control mechanisms entail a variety of actions (CR3) [10].

Consequently, a control instrument intends to make the complexity in which an organization acts more comprehensible. Due to the digital transformation, work has become more complex and the requirements for an effective control instrument have expended. Therefore, new control mechanisms ought to be more informal (CR4) and holistic (CR5) [10]. Cardinal et al. [10] state that these aspects have not been sufficiently addressed in control research.

Different approaches should be included to obtain a holistic control mechanism [10]. Due to the inherent information process properties of control mechanisms, information systems provide a suitable approach [37]. The man-technology-organization (MTO) model provides a fitting framework for this holistic viewpoint [38], as an information system represents a sociotechnical system consisting of humans and machines which create, use and process information. Thus, it visualizes a network of communication relationships between them, enabling them to describe complex organizational work systems within organizations [6]. Through the digital transformation, a control mechanism should therefore include the perspectives of people, technology and organization $[5,38]$.

In addition to a more holistic approach, controls ought to be informal, which refers "to unwritten mechanisms that influence employees by motivating them to enact accepted values, norms, and beliefs" [27]. Controlling these implicit rules is an important part of IOC, because they represent a prerequisite for effective collaboration [11]. The authors have identified "Linked to Business Objectives" (CR1), "Decision Support" (CR2), "Variety of Actions" (CR3), "Informal Control Elements" (CR4) and "Holistic MTO Based" (CR5) as requirements for an organizational control instrument for IOC, based on the findings of prevailing literature on IOC and control instruments.

In an initial literature review, different control instruments for intraorganizational collaboration were 
Table 1: Requirement analysis of control instruments for IOC

\begin{tabular}{|c|c|c|c|c|c|c|}
\hline Model & $\mathrm{CbM}[31]$ & TEaM [12] & 3DPMM [44] & ESSPAM [23] & SAMM [19] & WKCNM [36] \\
\hline $\begin{array}{c}\text { Underlying } \\
\text { Approach }\end{array}$ & $\begin{array}{c}\text { Transactive } \\
\text { Memory } \\
\text { Model }\end{array}$ & EFQM & $\begin{array}{c}\text { Performance } \\
\text { Measurement } \\
\text { Model }\end{array}$ & $\begin{array}{c}\text { Innovation } \\
\text { Diffusion, } \\
\text { Social Capital }\end{array}$ & $\begin{array}{c}\text { Maturity } \\
\text { Model }\end{array}$ & \begin{tabular}{|c} 
Knowledge \\
Collaboration \\
Network \\
\end{tabular} \\
\hline CR1 & 0 & 0 & $\bigcirc$ & 0 & 0 & ( \\
\hline $\mathrm{CR} 2$ & 0 & 0 & 0 & 0 & 0 & 0 \\
\hline CR3 & 0 & 0 & 0 & 0 & 0 & 0 \\
\hline CR4 & 0 & 0 & 0 & 0 & 0 & 0 \\
\hline CR5 & $\bigcirc$ & 0 & (1) & 0 & 0 & (1) \\
\hline
\end{tabular}

\begin{tabular}{|l|l|}
\hline Requirement is not or not enough taken into account & Requirement is fully or almost fully covered \\
\hline Requirement is partially taken into account & \\
\hline
\end{tabular}

identified [12, 19, 23, 31, 36, 44]. The models were analyzed regarding their consistency to the requirements CR1 to CR5 and the results are presented in Table 1. All models incorporate support of the decision process (CR2) as well as informal control elements (CR4). That all models contain the latter is related to the qualitative character of collaboration. However, the most significant challenge lies in Multifaceted Actions (CR3), which are not covered by any model and therefore ought to be considered in subsequent developments. Hence, no maturity model gives action plans on how to improve the analyzed elements. Although all models pursue the goal of improving intraorganizational collaboration, only the Situational Adaptable Maturity Model (SAMM) [19] and Weighted Knowledge Collaboration Network Model (WKCNM) [36] support alignment with the organization's strategy and goals (CR1). The Enterprise Social Software Platform Adoption Model (ESSPAM) [23] and SAMM already incorporate a holistic approach. The 3DPerformance Measurement Model (3DPMM) [44] and WKCNM cover the human and technological dimensions, with the former developed exclusively for collaboration in the design context within computeraided design (CAD) platform.

The Cognitive-Based Metrics Model (CbM) [31], teamwork excellence modified model (TEaM) [12] as well as the 3DPMM can not fully satisfy over half of the requirements and are therefore not suitable as an organizational control of IOC. The ESSPAM, SAMM and WKCNM meet most of the criteria and are therefore suitable for organizational control of IOC and further development. Nevertheless, a study from 2016 shows that only $13 \%$ of the surveyed organizations use control instruments for IOC at the organizational level [4]. To achieve the use of organizational control instruments for IOC, it is crucial to take requirements from a practical point of view into consideration and develop a practical requirement catalog.

Compared with the ESSPAM and WKCNM, we consider the SAMM as the most promising approach, as it provides the most detailed information about different improvement areas and therefore a good basis for the main deficit of deriving a variety of actions (CR3). Furthermore, the SAMM, with an underlying MM approach, is the only model, that meets the requirements of a holistic model (CR5) as well as the alienability with the organizational strategy and goals (CR1). Additionally, MMs are often used for the assessment of processes [30], which is consistent with the procedural character of IOC. The MM approach is "increasingly being applied within the field of information systems (IS) and management science" [30]. Due to the qualitative character of IOC, the result presentation as maturity levels provides a suitable visualization format that reduces biases caused by explicit numerical values. As the SAMM is adaptable to different situations, it addresses the disadvantage of a generic model $[25,19]$. Although the adaptation of MM to new conditions is costly and the assessment requires a high resource input [25], we consider MM as an applicable approach and hereafter this paper focuses exclusively on the MMapproach for the analysis of the practical requirements.

A MM "consists of a sequence of maturity levels for a class of objects. It represents an anticipated, desired, or typical evolution path of these objects shaped as discrete stages. Typically, these objects are organizations or processes" [3]. Therefore, MMs enable organizations, in regard to the model domain, in this case IOC, to position themselves, identify development perspectives and to improve it $[30,33]$. The maturity stages are sequential and define the current state of maturity in the model domain, whereby those stages are defined by a set of measurement criteria $[14,30]$. 


\section{Research Design}

This paper aims to answer the previously presented two research questions. Accordingly, the research design is structured in two sections. Firstly, in-depth interviews were conducted, from which a practical requirement catalog for an organizational control instrument for IOC was derived using a qualitative content analysis. Secondly, MMs for collaboration were identified and analyzed regarding the fit of the developed requirement profile using a systematic literature review.

\subsection{In-depth Interview}

The in-depth interview conducted with field experts is one of the most important research methods for the collection of qualitative data [24]. In-depth interviews following Boyce and Neale [9] were conducted, with a total of six experts to obtain an adequate sample size. The first step was to define what an expert is, by establishing mandatory and optional traits of an expert [9], as shown in Table 2. Two-thirds of the interviewed experts fulfill the optional traits and have experience in the implementation of IOC and/or knowledge about the assessment of efficiency and effectivity of IOC.

Table 2: Mandatory and optional traits for expert's selection

Mandatory Expert Traits

- connection to IOC in everyday work from a strategic level as well as the user perspective

- know-how about evaluation methods of physical collaboration and work processes

- working or consulting large or medium-sized organizations

\section{Optional Expert Traits}

- experience in the implementation of IOC

- knowledge to evaluate the effectiveness and efficiency of IOC

To capture the opinions and views of the experts a questionnaire guideline was developed in the second step [9], to provide the interviewers with an orientation framework for the interviews. The developed questionnaire was based on the theoretical background on IOC as well as organizational control and follows the structuring approach as suggested by Qu \& Dumay [34].

In the third step, the interviews were conducted virtually and varied between forty-five and sixty minutes. At least two out of three interviewers took part in every interview to receive different perspectives and to minimize the bias of the outcomes [34]. In the introductory part of the interviews, the interviewers explained the research process as well as the understanding of IOC in the context of this research to gain a common understanding as a basis for the interview. To provide the best possible comparability, all areas of the interview guideline were carried out, independently of its structure [13]. The final step was analyzing the collected data before disseminating the result. Therefore, all interviews were fully transcribed following the process as recommended by Kowal \& O'Connell [22] and subsequently analyzed.

To this end, a qualitative content analysis was carried out. As the material for the qualitative content analysis is taken from an open interview, explanations sometimes deviate from the central topic or relevant content occurs at different points in the material [29]. To use an effective, efficient and specific procedure, an inductive category assignment was carried out with MAXQDA according to Mayring [29]. The aim was to gain a summary of categories that were derived from the material itself and not from theoretical considerations [29]. These categories were used to finally develop a practical requirement catalog and answer the first research question.

\subsection{Systematic Literature Analysis}

To elaborate on the second research question, a systematic literature analysis in accordance with vom Brocke [42] was carried out. To identify relevant MMs the step of the literature search was conducted following Webster and Watson [43]. The search was limited to peer-reviewed articles in the academic databases "Business Search Complete", "Web of Science" and "ScienceDirect" that were published until December 2019. To achieve an effective search string, synonyms and related terms were obtained by means of a thesaurus. The database papers were searched by title, abstract and keywords for the search string: ("collaboration" OR "teamwork" OR "group work") AND "maturity model".

Table 3: Literature Search

\begin{tabular}{lcc}
\multicolumn{1}{c}{$\begin{array}{c}\text { Database/ } \\
\text { Search step }\end{array}$} & $\begin{array}{c}\text { Number of } \\
\text { results }\end{array}$ & $\begin{array}{c}\text { Number of } \\
\text { relevant } \\
\text { results }\end{array}$ \\
Business Search Complete & 26 & 2 \\
Web of Science & 80 & 4 \\
ScienceDirect & 23 & 1 \\
\hline Duplicates & $\mathrm{n} / \mathrm{a}$ & 3 \\
For-/Backward search & $\mathrm{n} / \mathrm{a}$ & 1 \\
\hline \hline Total & 129 & 5 \\
\hline \hline
\end{tabular}

Firstly, the results were screened regarding their topic relevance. Secondly, the relevant articles were checked for duplicates and extended by a forward as well as backward search [43]. As shown in Table 3, in 
the initial search 129 papers were found, with four relevant for IOC. Furthermore, one additional paper was identified through the forward and backward search.

In the final step, the results from the interviews and the literature search were combined. Hereby the MMs were examined regarding their goodness of fit as an organizational control instrument for IOC, in reference to the developed practical requirement catalog.

\section{Findings}

An outcome of the qualitative content analysis shows the relevance of an organizational control instrument for IOC. Another result of the interviews is a requirement catalog for such instruments, as shown in Table 4. Furthermore, as a result of the literature analysis, Table 5 shows to what extent the identified MMs for collaboration meet the practical requirements.

\subsection{Relevance}

As presented in section one, the first aspect to investigate is whether there is a need for a control instrument for IOC from a practical perspective. All experts confirmed, the importance of an effective instrument to control an organizations ability to collaborate as well as their corresponding facilitating actions. The interviewed experts all argued that processes for IOC, in their experience, often are solely based on observations and platform data such as user numbers, number of communities and platform groups as well as the number of chat and thread messages. Therefore, organizational control instruments for IOC are of relevance and the identified gap also exists from a practical point of view.

\subsection{Requirement Catalog}

To cluster the inductively derived requirements a classification for model development from de Bruin et al. [14] was used. Accordingly, the requirements are divided into three main categories: requirements regarding the model architecture, model application and model content [14]. Table 4 shows the requirement catalog with the identified requirements and their respective categories. An organizational control instrument must fulfill the different requirements and analyzing to what extent the requirements are met, indicates how practically applicable the instrument is. This allows a comparison between different approaches and models. Following, the different requirements (R) are described in detail and compared to the theoretical ones.
The model architecture is characterized by the requirements $\mathrm{R} 1$ to $\mathrm{R} 4$. The model architecture contains the structure of the model and must balance the complex reality with the desired as well as the understandable simplicity of a model. It forms the model basis and creates a framework in which the model can be changed and adapted [14].

Table 4: Requirement Catalog

\begin{tabular}{|c|c|c|}
\hline Type & ID & Requirement \\
\hline \multirow{6}{*}{$\begin{array}{l}\text { Model } \\
\text { Architecture }\end{array}$} & $\mathrm{R} 1$ & Adaption to the organization \\
\hline & $\mathrm{R} 2$ & Improvement potential \\
\hline & R3 & Derivation of actions \\
\hline & R4 & Targeted data collection \\
\hline & $\mathrm{R} 4.1$ & Datatype \\
\hline & $\mathrm{R} 4.2$ & Multiperspectivity \\
\hline \multirow{3}{*}{$\begin{array}{c}\text { Model } \\
\text { Application }\end{array}$} & R5 & $\begin{array}{l}\text { Classification by Reference } \\
\text { Guide }\end{array}$ \\
\hline & R6 & $\begin{array}{l}\text { Intraorganizational } \\
\text { comparison }\end{array}$ \\
\hline & R7 & Targeted result presentation \\
\hline $\begin{array}{l}\text { Model } \\
\text { Content }\end{array}$ & $\mathrm{R} 8$ & Holistic approach \\
\hline
\end{tabular}

Adaption to the organization (Rl)

Three of the experts mentioned the differences between organizations and the associated need for an adaption to the organizational specific circumstances. For example, organizations differ in their size, field of action, and organizational objectives. For this reason, an evaluation tool for IOC needs to take these differences into account and indicate varying characteristics. Hereby describes one expert the definition of success as important for the model content, as different aspects can be of importance when the organization's objectives vary. Consequently, to reach practical relevance, the model must be adaptable to these different characteristics and thus, R1 refers to the organizational adaption of the model content. This is similar to CR1, the link to business objectives, but exceeds this by including the general adaptability to company specifics. Improvement potential (R2)

To create added value for the organization, the instrument intends to systematically identify potential for improvement within the organization. Three experts pointed out, that the identified weaknesses are an opportunity to increase the efficiency and effectiveness of IOC if the right conclusions are drawn. The control instrument therefore ought to not only measure, but also provide guidance on how to interpret the result. This corresponds to the theoretical requirement CR2.

Derivation of actions ( $R 3$ )

This requirement is closely related to the previous one, as not only the identification of areas for improvement is important, but also actions to achieve 
them. Therefore, actions must be derived from the identified problem and improvement areas. As mentioned by five experts, the control instrument should not only identify the problem areas but also propose concrete actions and thereby support the process of continuous improvement. Therefore, the model needs to derive actions from the identified potentials. The theoretical requirement CR3 is similar to this, with the difference that the focus is on diversity versus specificity of actions.

\section{Targeted data collection (R4)}

The interviews indicate that a control instrument for IOC needs to fulfill several factors concerning what kind of data should be collected and used in the model. To this end, data subject groups must be identified. This requirement is closely related to the model content, as the content and corresponding data may vary from data subject to data subject. Since the collected data represents the reality basis of the model, it is an essential part of the model architecture.

R4.1 Similar to CR4, all experts explained that different types of data, both quantitative and qualitative, must be considered. Qualitative data refers to e.g. employee satisfaction concerning virtual collaboration or the implementation of technical support systems. Quantitative data, on the other hand, refers e.g. to the number of clicks or user activity.

$R 4.2$ Furthermore, five experts pointed out that a multi-perspective approach must be chosen. Accordingly, depending on the data subject, different data must be collected. On one hand, the data must represent different perspectives on an aspect, e.g. the analysis should cover both data from a strategic as well as an operational perspective. On the other hand, especially qualitative data must represent different perspectives on a data subject, e.g. how a data subject perceives a relevant aspect and how it is perceived by others.

The model application comprises R5 to R7. The model must be considered completely and accurately regarding the defined scope of application, to ensure the applicability and reliability of the model [14]. Therefore, the requirements represent the later user and usage perspective.

\section{Classification by Reference Guide (R5)}

All six experts said that in addition to identifying optimization potentials, the current degree of implementation should also be examined. Thus, the model should provide a classification system for the user and clarify the current situation in the organization. For example, a maturity level for the organization can be determined as a result. This is related to the identification of improvement potential (R2), as it provides guidance on how to interpret the result.
Furthermore, it offers a basis for the intraorganizational comparison described in R6.

Intraorganizational comparison (R6)

In addition to the classification, the instrument must also assess the results of the analysis in intraorganizational relation. Hence, a temporal comparison, as well as an interdepartmental comparison, should be made within the organization. In the temporal comparison, the same team or target group is considered at two different points in time, and conclusions are drawn about the success of the IOC. In contrast, the intra-departmental comparison considers two different teams (preferably with similar general conditions) at the same time. Based on this, room for improvement for each team is identified.

Targeted presentation of results $(R 7)$

The last requirement regarding the model application relates to the way the data and the results identified are presented. These should be made available to the user in a structured and user-oriented form. All experts stated to use a form of dashboard to visualize the results. The dashboard has the advantage to get all important information briefly and the user gets a quick overview.

Finally, the model content that must be considered is described by $\mathrm{R} 8$. The individual characteristics that have an impact on the maturity level are identified and defined here. This may have to be done at various levels of detail. In a multidimensional analysis, in which several different characteristics are considered, it is important to ensure that these characteristics provide a coherent picture with as little overlap as possible [14].

Holistic approach (R8)

Five experts emphasized that the content of the model is decisive for the value of the control instrument and that various aspects must be covered, as stated in the theoretical requirement CR5. Consequently, a holistic view has to be taken and as described in $\mathrm{R} 1$, the differences between organizations must be considered. In the interviews, various factors regarding the model content were identified, which should be considered when analyzing an organization's IOC. As single model contents were not part of the research objective, they have been excluded from this paper and should be part of a deeper investigation in further research. However, the interview findings support the theoretical considerations of section two, that the content factors can be divided into three main categories: Human, Technology and Organization.

\subsection{Maturity Model Analysis}

The requirement catalog is the basis for further analysis and demonstration of its applicability, allowing an analysis of existing models concerning the 
Table 5: Analysis of MMs for collaborative work regarding the practical requirement catalogue

\begin{tabular}{|c|c|c|c|c|c|c|c|}
\hline Type & & Requirement & CollabMM [28] & Col-MM [7] & $\mathrm{ECMM}[1]$ & VTMM [16] & SAMM [19] \\
\hline \multirow{5}{*}{ 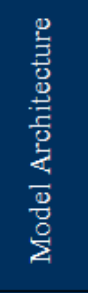 } & \multicolumn{2}{|c|}{ Adaption to organization } & O & (1) & (1) & O & O \\
\hline & \multicolumn{2}{|c|}{ Improvement potential } & 0 & 0 & 0 & O & O \\
\hline & \multicolumn{2}{|r|}{ Derivation of actions } & (1) & O & O & (1) & (1) \\
\hline & \multirow{2}{*}{$\begin{array}{c}\text { Targeted } \\
\text { data } \\
\text { collection }\end{array}$} & Datatype & $\bigcirc$ & 0 & $\bigcirc$ & $\bigcirc$ & $\bigcirc$ \\
\hline & & Multiperspectivity & $\bigcirc$ & ○ & (1) & $\bigcirc$ & (1) \\
\hline \multirow{3}{*}{ 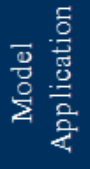 } & \multicolumn{2}{|c|}{ Classification by Reference Guide } & (1) & 0 & 0 & O & ? \\
\hline & \multicolumn{2}{|c|}{ Intraorganizational comparison } & O & O & O & (1) & $\bigcirc$ \\
\hline & \multicolumn{2}{|c|}{ Targeted result presentation } & O & O & O & $\bigcirc$ & O \\
\hline \multirow{3}{*}{ 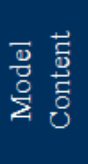 } & \multirow{3}{*}{$\begin{array}{l}\text { Holistic } \\
\text { approach }\end{array}$} & Human aspects & $\bigcirc$ & $\bigcirc$ & $\bigcirc$ & O & \\
\hline & & Technological aspects & $\bigcirc$ & O & 0 & (1) & O \\
\hline & & Organizational aspects & (1) & (1) & 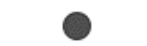 & $\bigcirc$ & \\
\hline
\end{tabular}

\begin{tabular}{|ll|c|}
\hline & Requirement is not or not enough taken into account & Requirement is fully or almost fully covered \\
\hline & Requirement is partially taken into account &
\end{tabular}

fulfillment of these requirements. As described in section two, MMs are considered an applicable approach and are subject to further analysis.

For this purpose, five MMs were identified in the literature search and are subject to the analysis. Requirements that were not described in the models were interpreted as "requirement is not or not enough taken into account". The results are presented in Table 5 and are discussed in the next section.

\section{Discussion}

The preceding comparison of the five maturity models with relation to IOC as shown in Table 5, shows that every maturity model has different strengths and weaknesses with respect to the IOC context.

The Collaboration Maturity Model for Business Processes (CollabMM) [28] fulfills the requirements of identifying improvement potential as well as intraorganizational comparison. For the derivation of actions, the CollabMM offers suggestions. However, the suggested actions are not sufficient for the implementation in organizations concerning their scope and variety. The classification by reference guide is partially considered. A holistic approach with the used definition is not possible with the CollabMM because only organizational aspects get noticed partially. Furthermore, lacks this MM in the area of data collection as none of the requirements are met. An adaption to the specific organization is not possible and a structured presentation view is not mentioned. This $\mathrm{MM}$ focuses on collaboration processes and neglects many requirements that have to be met in the context of a comprehensive model for IOC.

In contrast to the CollabMM the Collaboration Maturity Model (Col-MM) [7] shows strengths in the data collection as all requirements are at least partially met. Another advantage of the Col-MM is, the at least partially fulfilled adaption to the organization. Also, the targeted presentation of results is part of the Col-MM. Furthermore, the derivation of actions is intended in this maturity model, but it does not contain any proposed action, which is the most common and biggest criticism of existing MM in general [25, 30]. As well as the CollabMM the Col-MM does not include cultural nor technological aspects. Boughzala \& DeVreede [7] also state that the definition of maturity levels is not sufficient to implement the Col-MM and needs further development. Besides the insufficient maturity levels, the Col-MM has a strong focus on the applicability.

Similar to the already mentioned maturity models the Enterprise Collaboration Maturity Model (ECMM) [1] supports the identification of potential for improvement. However, the derivation of actions is intended but as well as the Col-MM no suggestions for actions or a process on how to derive actions are mentioned. The ECMM has some weaknesses in the data collection because the evaluation of quantitative and qualitative data is not part of the maturity model and the multi-perspective approach and the definition of target groups are only partially considered. Also, an intraorganizational comparison is not intended. But the ECMM offers a stronger holistic approach than the CollabMM and the Col-MM. As the CollabMM and the 
Table 6: Comparison of MMs by model area regarding suitability for further MM development

\begin{tabular}{|c|c|c|c|c|c|}
\hline Model & CollabMM [28] & Col-MM [7] & ECMM [1] & VTMM [16] & SAMM [19] \\
\hline Model Architecture & no & yes & partially & no & yes \\
\hline Model Application & partially & yes & partially & partially & partially \\
\hline Model Content & no & no & partially & partially & yes \\
\hline
\end{tabular}

\begin{tabular}{|c|c|c|c|}
\hline yes & suitable as basis & partially & partially suitable as basis \\
\hline no & not suitable as basis & & \\
\hline
\end{tabular}

Col-MM, the ECMM focuses on organizational aspects, but in contrast this MM considers the technological perspective as well.

Friedrich et al. [16] focus strongly on the interpersonal process of virtual work in a team setting. The Virtual Team Maturity Model (VTMM) [16] is a rather practical approach to control a virtual team by proposing best practices concerning team tools and culture. As its strength is in the best practice database for the derivation of actions, it provides a valuable tool for leaders of distributed and virtual teams. The specialization on individual virtual teams presumably makes this maturity model not suitable as an organizational control instrument for an overall assessment of the organization, as it doesn't provide the complexity. Another drawback is the lack of approaches of data collection on how to make the success factors of IOC measurable as well as the possibility to adapt the model to organizational specifics. Compared to the ECMM it focuses mainly on cultural aspects instead of organizational but includes the technological perspective as well, although only partially.

The SAMM by Jansz [19] fulfills a wide array of the identified criteria. Especially the holistic view on the success factors, adaptability to organizational specifics and the identification of potential for improvement is making this model a very comprehensive and detailed model. In contrast to the other considered models, it is the only one that covers the adaptation to the organization almost fully. Nevertheless, there are several limitations to the model. In the empirical evaluation of the maturity model drawbacks concerning the understandability and reliability became apparent. In general, it is predominantly theory-based which leads to a high complexity that could be cumbersome when implementing it in a practical setting. In the assessment of the model, it was also found that it presumably would not support the acceptance and communication of changes. The major drawback, that follows the theory focus model is the lack of measurability of actions from the improvement areas and derivation of concrete actions.

As the previous analysis of the different MMs presents, none of the selected models meets all requirements nor is ready to be used in organizations. However, the MMs have various approaches with different strengths and weaknesses, which therefore provide a good basis for further development. To give recommendations for selecting one or several suitable models for further development, the results were combined using the three model development categories architecture, application and content. The results of this comparison are shown in Table 6. Although the CollabMM and VTMM don't provide a suitable model architecture, they address the general MM problem of deriving actions. Compared to the CollabMM, the VTMM includes 2 different content perspectives and shows overall a more complex structure. Therefore, both models should be at least looked into regarding this general problem area of MMs. Juxtaposing the benefits and limitations of the SAMM, it provides the most complex model and primary lacks in its applicability and reliability. Accordingly, the authors consider the SAMM to be the most suitable option for further development. This should involve further research into the derivation and evaluation of actions (R3), the expansion of the data collection (R4), intraorganizational comparison (R6) as well as the underlying success factors (R8).

\section{Conclusion}

This paper illustrates the theoretical and practical relevance of an organizational control instrument to design and maintain IOC. Furthermore, a requirement catalog for such instruments is presented and its applicability in the context of MMs for IOC is demonstrated. The findings show that although suitable MM-approaches exist in the domain of IOC, they can still be improved and need further development.

To answer the first research question, the requirements for such an instrument were identified to evaluate the extent to which existing approaches meet and lack the practical requirements. The outcomes demonstrate that there are eight different requirements that an instrument ought to meet in order to be sufficiently practical. These requirements are categorized into the model's architecture, application as 
well as content and form the intended requirement catalog. Comparing the theoretical and practical requirements, several parallels can be drawn. Both show, for instance, that a model should support the decision process (CR2 and R2) and be holistic (CR5 and $\mathrm{R} 8$ ). Still, the practical requirement catalog is more complex.

To answer the second research question, this catalog was applied to the MM context, comparing them regarding the fulfillment of the requirements. The results show that no identified $\mathrm{MM}$ in the literature fulfils all requirements. The MMs show different strengths and weaknesses and can be used for further developments to gain a more suitable MM for IOC.

Based on the results as shown in Table 5, the SAMM model fulfills the most requirements in comparison to the other models. It is the only model, that incorporates a holistic perspective and provides an adaptation possibility to the organization. As it lacks primarily in its applicability, we can consider the best suitable basis for further development, from the analyzed MMs. We propose to update the underlying success factors and enrich them with quantitative indicators, to minimize the self-evaluation bias. Furthermore, we propose to develop a procedure to derive and evaluate adequate actions, as a general problem of MMs is the derivation of actions [25] and a major one of the SAMM as well.

This paper has theoretical implications for distributed teams and organizations. The results of this paper increase the understanding of what aspects are relevant for controlling distributed teams and organizations with respect to IOC. Furthermore, the outcomes present a starting point for further developments of an MM approach for IOC. From this point, future research should focus on how the analyzed MMs can be used as a basis and adapted to be practical, reliable and value adding.

It should be considered that the paper focuses exclusively on intraorganizational collaboration in an online context. Further research should focus on links between other kinds of collaboration and their requirements. As this study used only a qualitative approach, the validity of the requirement catalog should be confirmed by a quantitative study considering organizations that differ in e.g. size, industry and nationality. In addition, the requirement profile requires further investigations since the model content is only considered on an abstract level. Further research should therefore investigate what specific content a model should include in the three model content areas man, organization and technology. The derivation of actions should be an investigation focus in further $\mathrm{MM}$ developments as it is a major problem of the model architecture.

\section{References}

[1] Alonso, J., Martínez de Soria, I., Orue-Echevarria, L. and Vergara, M., "Enterprise Collaboration Maturity Model (ECMM): Preliminary Definition and Future Challenges", Enterprise Interoperability IV, Springer, London, 2010, pp. 429-438.

[2] Azab N. A., Cases on Web 2.0 in developing countries: Studies on implementation, application, and use: Studies on implementation, application, and use. IGI Global, Hershey, 2012

[3] Becker, J., Knackstedt, R. \& Pöppelbuß, J., Developing maturity models for IT management - a procedure model and its application, Bus. Inform. Syst. Eng. 1 (2009) 213-222.

[4] Beezy, Measuring Collaboration Success: Community Survey Results on Defining Successful Collaboration, 2016.

[5] Bockshecker, A., S. Hackstein, and U. Baumöl, "Systematization of the term digital transformation and its phenomena from a socio-technical perspective - A literature review", Twenty-Sixth European Conference on Information Systems (ECIS), Portsmouth, United Kingdom, 2018.

[6] Bostrom, R.P. and J.S. Heinen, "MIS Problems and Failures: A Socio- Technical Perspective Part I: The Causes“, MIS Quarterly, 1(September), 1977, pp. 17-32.

[7] Boughzala, I. and de Vreede, G.J., "A collaboration maturity model: development and exploratory application", In: HICSS '12: 45th Hawaii International Conference on System Science, Maui, Hi, United States. 2012, pp. 306 - 315.

[8] Bouras, C., Giannaka, E. and Tsiatsos, T., "Designing virtual spaces to support learning communities and ecollaboration", In: Fifth IEEE International Conference on Advanced Learning Technologies (ICALT'05), Kaohsiung, 2005, pp. 328-332.

[9] Boyce, C., and P. Neale, Conducting in-depth interviews: A guide for designing and conducting in-depth interviews for evaluation input, Pathfinder International Tool Series, 2006.

[10] Cardinal, L. B., Kreutzer, M., and Miller, C. C., “An aspirational view of organizational control research: Reinvigorating empirical work to better meet the challenges of 21 st century organizations", Academy of Management Annals, 11(2), 2017, pp. 559-592.

[11] Cardon P.W., "Community, culture, and affordances in social collaboration and communication", International Journal of Business Communication, 53(2), 2016, pp. 141147.

[12] Castka, P., Bamber, C.J. and Sharp, J.M., "Measuring teamwork culture: the use of a modified EFQM model", Journal of Management and Development, 22(2), 2003, pp. 149-170.

[13] Creswell, J.W., Qualitative Inquiry \& Research Design. Choosing Among Five Approaches, 2nd Edition, Sage Publications, Thousand Oaks, 2007.

[14] De Bruin, T., M. Rosemann, R. Freeze and U. Kulkarni, "Understanding the main phases of developing a maturity assessment model", In: Proceedings of the 16th Australasian Conference on Information Systems (ACIS), Sydney, 2005, pp. 8-19. 
[15] Downes, R., "The Proximity Paradox: How Distributed Work Affects Relationships and Control", Proceedings of the 53rd Hawaii International Conference on System Sciences, 2020, pp. 440-449.

[16] Friedrich, R., Bleimann, U., Stengel, I. and Walsh, P., "VTMM - Virtual Team Maturity Model", In: Proceedings of the 7th European Conference on Management, Leadership and Governance: SKEMA Business School, Sophia-Antipolis, France, C. Despres, Ed, Reading: Academic Publishing, 2011, pp. $159-166$.

[17] Haythornthwaite, C., "Facilitating collaboration in online learning", Journal of Asynchronous Learning Networks, 10(1), 2006, pp. 7-24.

[18] Holsapple, C.W. and Joshi, K.D., "An investigation of factors that influence the management of knowledge in organizations", Journal of Strategic Information Systems, 2000, pp. 235-61.

[19] Jansz, S., Corporate Collaboration 2.0 Maturity Model. Shaker Verlag GmbH, Aachen, Germany, 2016.

[20] Kirsch, L. J., "The management of complex tasks in organizations: Controlling the systems development process", Organization science, 7(1), 1996, pp. 1-21.

[21] Kock, N., "Designing E-Collaboration Technologies to Facilitate Compensatory Adaptation", Information Systems Management, 25(1), 2008, pp. 14-19.

[22] Kowal, S. and O'Connell, D.C., "The Transcription of Conversations", In: Flick, U., v. Kardoff, E. and Steinke, I. (eds.), A Companion to Qualitative Research (248), Sage, London, Thousand Oaks, New Delhi, 2004.

[23] Kügler, M., Smolnik, S., and Raeth, P., "Determining the factors influencing enterprise social software usage: Development of a measurement instrument for empirical assessment", In 2013 46th Hawaii International Conference on System Sciences, 2013, pp. 3635-3644.

[24] Kvale, S., and Brinkmann, S., InterViews: Learning the Craft of Qualitative Research Interviewing, 2nd Edition, Sage, Los Angeles, CA, 2009.

[25] Lasrado, L., Vatrapu, R., Karsgaard, H. B., and Kjaer, J. F., "Towards sustainable design for maturity measurement marketplace", Information Systems and Management in Creative eMedia, 2016 (2), pp. 25-33.

[26] Lepsinger, R., "The virtual challenge: It's more than cultural differences", People \& Strategy, 35(1), 2012, pp. 10-11

[27] Long C.P. and S.B. Sitkin, "Control-trust dynamics in organizations: identifying shared perspectives and charting conceptual fault lines," Academy of Management Annals, 12(2), 2018, pp. 725-751.

[28] Magalhãe Magdaleno, A., Cappelli, C., Araujo Baião, F., Maria Santoro, F. and Araujo, R., "Towards Collaboration Maturity in Business Processes: An Exploratory Study in Oil Production Processes", Information Systems Management, 25(4), 2008, pp. 302-318.

[29] Mayring, P., Qualitative content analysis: theoretical foundation, basic procedures and software solution, Klagenfurt, 2014.

[30] Mettler, T., "Maturity assessment models: a design science research approach", International Journal of Society Systems Science (IJSSS), 3(1/2), 2011, pp. 81-98.
[31] Noble, D., and M. Letsky, "Cognitive-based metrics to evaluate collaboration effectiveness", 2003.

[32] Peffers, K., Tuunanen, T., Rothenberger, M.A. and Chatterjee, S, "A Design Science Research Methodology for Information Systems Research", Journal of Management Information Systems, 24(3), 2007, pp. 45-77.

[33] Pöppelbuß, J. and Röglinger, M., "What Makes a Useful Maturtiy Model? A Framework of General Design Principles for Maturity Models and its Demonstration in Business Process Management", In: ECIS 2011 Proceedings, Paper 28, 2011.

[34] Qu, S.Q. and Dumay, J., "The qualitative research interview", Qualitative Research in Accounting \& Management, 8(3), 2011, pp. 238-264.

[35] Schrage, M., Shared minds: The new technologies of collaboration, Random House, New York, 1990.

[36] Su, J., Y. Yang and N. Zhang, "Measurement of knowledge diffusion efficiency for the weighted knowledge collaboration networks", Kybernetes, 46(4), 2017, pp. 672692.

[37] Turner, K. L., and M.V. Makhija, "The role of organizational controls in managing knowledge", Academy of management review, 31(1), 2006, pp. 197-217.

[38] Ulich, E., Man- technology- organization: An European production concept. Betonwerk und FertigteilTechnik/Concrete Precasting Plant and Technology, 65(2), 1999, 26-31.

[39] Van Wart, M., A. Roman, X. Wang, and C. Liu, "Operationalizing the definition of e-leadership: identifying the elements of e-leadership", International Review of Administrative Sciences, 85(1), 2019, pp. 80-97.

[40] Verburg, R.M., A.M. Nienaber, R.H. Searle, A. Weibel, D.N. Den Hartog, and D.E. Rupp, "The role of organizational control systems in employees' organizational trust and performance outcomes", Group \& organization management, 43(2), 2018, pp. 179-206.

[41] Vogel, P. and Hultin, G., "Introduction: Digitalization and Why Leaders Need to Take It Seriously", Conquering Digital Overload, Springer International Publishing, 2018, pp. 1-8.

[42] Vom Brocke, J., A. Simons, B. Niehaves, B. Niehaves, K. Reimer, R. Plattfaut, and A. Cleven, "Reconstructing the giant: On the importance of rigour in documenting the literature search process", 17th European Conference on Information Systems (ECIS), Verona, 2009, pp. 2206-2217.

[43] Webster J. and R. T. Watson. "Analyzing the past to prepare for the future: Writing a literature review", MIS quarterly, 2002, pp. xiii-xxiii.

[44] Yin, Y., S. Qin, and R. Holland, "Development of a project level performance measurement model for improving collaborative design team work", In 2008 12th International Conference on Computer Supported Cooperative Work in Design, 2008, pp. 135-140.

[45] Zhao, Y., "Virtual Experience Is Real but Not Actual", Journal of Political Science and Public Affairs, 2(2), 2014, pp. 117-119. 